\title{
Oxygen isotopes in the solar system
}

\author{
TREVOR R. IRELAND ${ }^{1}$
}

${ }^{1}$ Research School of Earth Sciences, The Australian

National University, Canberra, ACT2601, Australia

(trevor.ireland@anu.edu.au)

Predissociation and self-shielding of carbon monoxide by UV radiation provides a strong astrophysical context for the origin of oxygen isotopic variations in the solar system. Enrichments in ${ }^{17} \mathrm{O}$ and ${ }^{18} \mathrm{O}$ can be related to shielded regions of a molecular cloud or nebula where the photons with energy required for $\mathrm{C}^{16} \mathrm{O}$ dissociation have been attenuated, but photons capable of breaking $\mathrm{C}^{17} \mathrm{O}$ and $\mathrm{C}^{18} \mathrm{O}$ are available. Observations that support this concept are: Sun has the most ${ }^{16} \mathrm{O}$-rich composition of the main reservoirs in the solar system, and, ${ }^{17} \mathrm{O}-{ }^{18} \mathrm{O}-$ rich compositions have been measured in various objects that suggest the presence of a heavy nebula water composition. The site of this fractionation is most reasonably associated with molecular clouds where evidence for this process was first observed.

While the components appear to be consistent with a number of observations of solar system materials, there are still a number of issues to address. How does the ${ }^{17} \mathrm{O}-{ }^{18} \mathrm{O}-$ rich water survive mixing and thermal processing with the indigenous water of the solar system? As dust spirals inward through the nebula, evaporation of the water will occur removing, or at least diminishing, the signature of the nebula water.

The oxygen isotopic compositions of the inner planetary system (as reflected in Earth, Mars, Vesta, chondrites) are systematically enriched in ${ }^{17} \mathrm{O}-{ }^{18} \mathrm{O}$ relative to Sun. This requires substantial reaction and homogenisation of the inner planetary system through reaction between primordial oxide/silicate minerals (of solar oxygen isotope composition) and the nebula water enriched in ${ }^{17} \mathrm{O}$ and ${ }^{18} \mathrm{O}$. A prediction of this model is that silicates on outer solar system bodies should reflect a primordial, solar, composition. This prediction could be tested by a remote sample analysis mission capable of high precision oxygen 3isotope analysis.

An alternative explanation would envisage that the average oxygen isotope composition of oxide/silicate dust coming in to the solar system is already close to the planetary composition. Reactions of that dust with nebula water and high temperature solar gas are then responsible for dispersion in oxygen isotopes. The corollary to this model is that the Sun has a composition that is more enriched in ${ }^{16} \mathrm{O}$-rich $\mathrm{CO}$ than is in equilibrium with the silicates. 